Arroyo-Vega, C., \& Lechuga-Granados, A. (2021).

Equinodermos de Isla La Roqueta de Acapulco,

Guerrero, México. Revista de Biología

Tropical, 69(S1), 265-271. DOI 10.15517/rbt.

v69iSuppl.1.46358

DOI 10.15517/rbt.v69iSuppl.1.46358

\title{
Equinodermos de Isla La Roqueta de Acapulco, Guerrero, México
}

\author{
César Arroyo-Vega ${ }^{1}$ \\ Adriana Lechuga-Granados*1
}

1. Facultad de Biología, Universidad Michoacana de San Nicolás de Hidalgo, Morelia, Michoacán, México; arroyoovegaa@gmail.com, adriana.lechuga@umich.mx (*Correspondencia).

Recibido 30-VI-2020. Corregido 28-X-2020. Aceptado 06-XI-2020.

\author{
ABSTRACT \\ Echinoderms from La Roqueta Island in Acapulco, Guerrero, México
}

\begin{abstract}
Introduction: Guerrero is the fourth most biodiverse state in Mexico, an example of this is the wealth of echinoderms that inhabit its coasts, it houses the largest number of species compared to the states of the Mexican South Pacific, it has 135 species, while Oaxaca 94 and Chiapas 15. The present study was developed on La Roqueta Island in Acapulco, Guerrero, Mexico, it is part of $0.3 \%$ of the national territory. The islands are the habitat of a greater number of species and endemisms, compared to continental areas of the same size, isla La Roqueta, like other islands in Mexico, are recognized as sites of great value, however, they face serious threats that put their stability at risk. Objective: To list the species of echinoderms on the island of La Roqueta de Acapulco, Guerrero, México. Methods: We collected in August and October 2018 and May 2019, through free diving and Scuba, at four sites, using direct capture, sweep search, random and transect techniques. Results: 214 specimens were collected, in four classes, nine orders, 16 families and 23 genera, for a total of 40 species. Important records are recognized: Ophiocoma sp., Phataria sp., Pentaceraster sp., Cucumaria sp., Astropyga sp., Meoma sp., Diadema sp., Afrocucumis ovulum, Pseudocnus californicus, Neocucumis veleronis and Holothuria imitans. Conclusions: The complexity of the various marine environments and substrates in Guerrero, particularly on La Roqueta Island, denotes the possibility of new species.
\end{abstract}

Key words: Echinodermata; richness; taxonomic listing; new registration; La Roqueta Island; Guerrero; México.

Los equinodermos son invertebrados deuterostomados marinos que se distinguen en las comunidades bentónicas como elementos esenciales, son parte importante de las cadenas tróficas, cumplen funciones como organismos depredadores, filtradores y detritívoros, su actividad contribuye al ciclo de nutrientes, a la descomposición de la materia orgánica, así como a la oxigenación de sedimentos y agua en los ecosistemas bentónicos marinos (Padilla-Pérez, Rodríguez-Troncoso, SoteloCasas, \& Cupul-Magaña, 2017). En México se encuentran 643 especies de equinodermos, siendo la Clase Ophiuroidea la más rica con 197 especies, Asteroidea contiene 185, Echinoidea 119, Holothuroidea 113 y Crinoidea 29 (Solís-Marín, Laguarda-Figueras, \& HoneyEscandón, 2014).

Guerrero es el cuarto Estado más biodiverso en México, incluyendo 930 especies de vertebrados en su territorio, pero sufre de una pérdida significativa de hábitats naturales originales. Así mismo, es uno de los Estados menos estudiados en México (Botello, 
Sánchez-Cordero, \& Ortega-Huerta, 2015). Aunado a los pocos estudios sobre su biodiversidad, en los últimos años Guerrero ha sido fuertemente influenciado por la inseguridad, generada por conflictos de narcotráfico y delincuencia organizada, lo cual ha causado que investigadores en diferentes áreas del conocimiento no lo consideren como sitio de estudio en sus proyectos de investigación. Bajo este escenario, se reconoce que en él habita el mayor número de especies de equinodermos en comparación con los estados del Pacífico Sur Mexicano. La valiosa aportación de la exhaustiva búsqueda de registros en literatura y de especímenes depositados en colecciones de referencia, da un listado de 162 especies válidas de equinodermos en el Pacífico Sur Mexicano incluidas en 96 géneros, 54 familias y 20 órdenes. El Estado de Guerrero registra 135 especies, Oaxaca 94 y Chiapas 15. La Clase Holoturoidea es la más diversa en Guerrero con 48 especies (Granja-Fernández, SolísMarín, Benítez-Villalobos, Herrero-Pérezrul, \& López-Pérez, 2015).

El Estado de Guerrero es considerado como región o sitio prioritario para la conservación de la biodiversidad en ocho categorías diferentes, las cuales, se distinguen por ser espacios geográficos con amplia variedad de ecosistemas que sustentan alta diversidad e integridad ecológica de especies nativas de flora y fauna, con especial importancia para su conservación (CONANP, 2018). Al suroeste del Estado se encuentra la Bahía de Acapulco, donde se localiza la isla La Roqueta. Esta isla, fue decretada como Parque Nacional Marino en 1982 bajo la vigilancia y resguardo de la Secretaría de Marina, el aspecto turístico quedó a cargo de la Secretaría de Turismo del Estado de Guerrero. A partir de 1999 ostenta la categoría de Unidad de Manejo Ambiental (UMA) coordinada entre las Direcciones de Turismo y Ecología del Municipio de Acapulco (Niño-Gutiérrez \& Pérez-Alcántara, 2011). Recientemente, algunos estudios manifiestan la necesidad de llevar a cabo acciones para proteger a la isla La Roqueta, y existe la propuesta de recategorizarla con carácter de
Área de Protección de Flora y Fauna (APFF) (Niño, 2010).

La importancia de las islas de México se reconoce por ser los territorios más ricos del mundo, la biodiversidad insular incluye a todos los ecosistemas, comunidades bióticas, especies, poblaciones y genes de las plantas $\mathrm{y}$ animales existentes, tanto en las porciones terrestres de las islas como en las aguas que las rodean, frecuentemente las especies insulares de flora y fauna, tanto terrestre como marina, incluyen endemismos únicos que evolucionaron en aislamiento de la biota continental, las islas de México son de gran importancia para la conservación de la biodiversidad nacional, ya que mantienen ecosistemas y especies endémicas que no ocurren en el territorio continental, pero también porque constituyen hábitats importantes para la alimentación, reproducción y refugio de muchas especies migratorias (Aguirre-Muñoz et al., 2010). En particular para la isla La Roqueta, Estado de Guerrero, son necesarios estudios que brinden un amplio panorama de aquellos factores que permiten la gestión ambiental de la isla, como sitio de importancia internacional, desde un enfoque turístico, así como desde el aspecto ecológico y biológico (Niño-Gutiérrez, 2008).

Algunos estudios sobre la riqueza, abundancia y diversidad de ofiuroideos, en cinco sitios rocosos de Acapulco, han registrado 11 especies de 1174 ejemplares recolectados. Particularmente se reconoce la presencia de seis especies de la Clase Ophiuroidea en 303 ejemplares recolectados en Palmitas, sitio localizado en la isla La Roqueta (Hernández, 2015). Un estudio similar sobre la Clase Ophiuroidea en cinco sitios de la Bahía de Acapulco, registró un total de 13 especies en 1908 ejemplares recolectados de ofiuroideos, donde Palmitas, uno de los cinco sitios estudiados en el área insular de La Roqueta se caracteriza por una franja rocosa de 5 a $10 \mathrm{~m}$ de profundidad con bajo oleaje, registrando en este último sitio una recolecta de 462 ejemplares y ocho especies de ofiuroideos (García, 2019).

El reconocimiento de la biodiversidad de equinodermos en La Roqueta como área 
insular, representa una zona importante en el Estado y en México, ya que cuenta con una compleja variedad de ambientes como arrecifes coralinos, fondos arenosos y rocosos, por lo que las contribuciones de este trabajo enriquecen el conocimiento sobre la riqueza de equinodermos, además, fortalece las estrategias de conservación y protección de estos peculiares hábitats.

\section{MATERIALES Y MÉTODOS}

Sitio de estudio: Isla La Roqueta se localiza al suroeste de la Bahía de Acapulco, Guerrero, México, (1649'30" - 16 49'02" N \& $99^{\circ} 54^{\prime} 03^{\prime}$ - $\left.99^{\circ} 55^{\prime} 07^{\prime \prime} \mathrm{W}\right), 120 \mathrm{msnm}$ y 75 ha. Vecinas a La Roqueta se encuentran el islote El Morro y la isla La Hierbabuena (Niño-Gutiérrez, 2008). Geomorfológicamente se ubica en la planicie aluvial de la Llanura Costera del
Océano Pacífico, con un clima subhúmedo con lluvias en verano, una oscilación térmica de 0.6 ${ }^{\circ} \mathrm{C}$ entre $26.5^{\circ} \mathrm{C}$ y $27.1^{\circ} \mathrm{C}$ y una temperatura media anual de $26.5^{\circ} \mathrm{C}$ (Niño-Gutiérrez, 2008).

El muestreo se llevó a cabo en cuatro sitios a lo largo de $1.4 \mathrm{~km}$ en la parte noroeste de la isla, lugares conocidos como Palmitas (1649'26.9" N \& 9954'44" W), El Rincón (1649'23" N \& 9954'48" W), Jardín $\left(16^{\circ} 49^{\prime} 23\right.$ " N \& 99 $\left.54^{\prime} 45^{\prime \prime} \mathrm{W}\right)$ y El Guano (16 49'25" N \& 99 55'7” W) (Fig. 1).

Trabajo de campo: Se realizaron recolectas en cada uno de los sitios en agosto y octubre 2018 y mayo de 2019. En total se efectuaron tres recolectas por sitio y doce inmersiones a lo largo de $1.4 \mathrm{~km}$. Además de los buceos libres en cada uno de los cuatro sitios del área de estudio, también se realizó buceo Scuba por dos o tres buzos con un tiempo de inmersión de

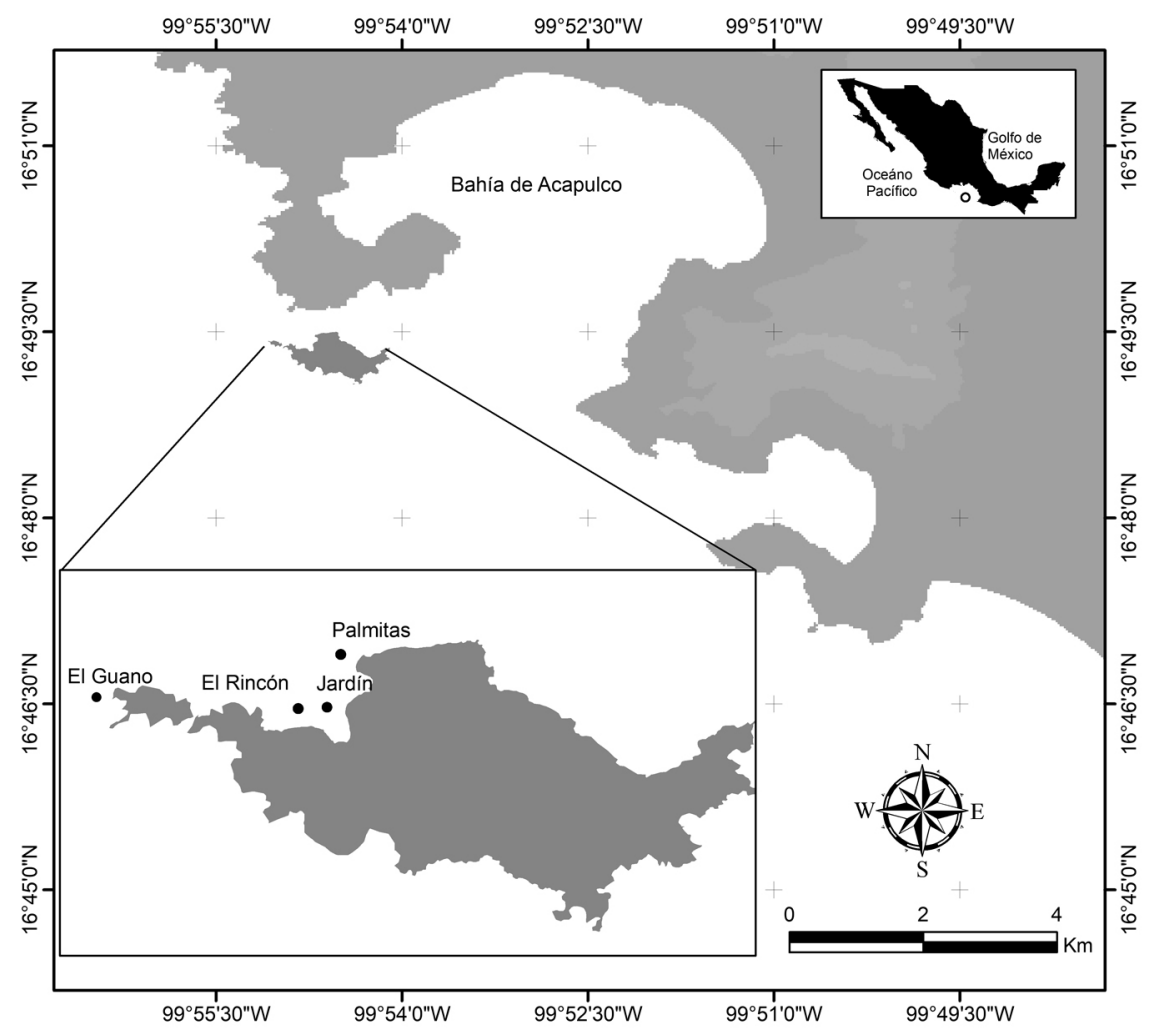

Fig. 1. Sitios de muestreo de equinodermos en isla La Roqueta, Acapulco, Guerrero, México. Fig. 1. Echinoderms sampling sites on La Roqueta island, Acapulco, Guerrero, México. 
50 min en promedio. Las técnicas de captura de equinodermos utilizadas fueron: colecta directa, búsqueda de barrido, aleatorio y transecto. Los organismos recolectados se narcotizaron con una solución de agua de mar y cloruro de magnesio $\left(\mathrm{MgCl}_{2}\right)$. Luego se realizó un registro fotográfico. Algunos organismos se fijaron en alcohol al $75 \%$ y algunos otros se conservaron en seco. Por último, se rotularon con datos de campo (fecha, localidad, profundidad, temperatura del agua y tipo de sustrato).

Trabajo de laboratorio: Se realizó el debido tratamiento de preservación y conservación de los ejemplares recolectados, en vía húmeda o seca. La identificación y elaboración del listado taxonómico de los ejemplares preservados, se llevó a cabo en las instalaciones de la Facultad de Biología de la Universidad Michoacana de San Nicolas de Hidalgo. La identificación de las especies recolectadas se realizó utilizando las claves taxonómicas de Caso (1951, 1961), Solís-Marín, ArriagaOchoa, Laguarda-Figueras, Frontana-Uribe, \& Durán-González (2009), Conejeros-Vargas (2015) y Granja-Fernández et. al., (2015).

\section{RESULTADOS}

Se recolectaron 214 ejemplares durante el trabajo de campo en agosto y octubre 2018 y mayo 2019. La recolecta se realizó en cada una de las tres visitas a la isla, cada sitio fue muestreado en tres ocasiones y en tres meses diferentes. Se realizaron 12 inmersiones con buceo libre y Scuba desde 0 a $23 \mathrm{~m}$ de profundidad, la temperatura del agua osciló entre los $26-31{ }^{\circ} \mathrm{C}$, en octubre del 2018 se registró la temperatura más baja del agua y en agosto del 2018 la temperatura más elevada. Se registró un total de 40 especies de equinodermos en la isla La Roqueta en Acapulco, siendo la Clase Ophiuroidea la más rica con 14 especies. Esta clase representó un $35 \%$ de las especies con respecto al resto de las clases, seguida de Holothuroidea (13 especies) (33\%), Echinoidea (ocho especies) $(20 \%)$ y Asteroidea (cinco especies) (12\%). El listado de equinodermos para la isla La Roqueta está integrado por nueve órdenes, 16 familias y 23 géneros. (Tabla 1). La familia con mayor representación de la Clase Ophiuroidea fue Ophiodermatidae (tres especies). En la Clase Holothuroidea la familia mejor representada fue Holothuriidae (cinco especies), seguida por Sclerodactylidae (cuatro especies). Las familias mejor representadas de erizos de mar son Diadematidae (dos especies) y Echinometridae (una especie). Las familias de estrellas de mar mejor representadas fueron Oreasteridae y Ophidiasteridae con dos especies cada una. El recuento total de las especies registradas en el presente estudio incluye a nueve unidades taxonómicas reconocibles denominadas morfoespecies, seis para la Clase Ophiuroidea y tres para la Clase Holothuroidea. Tripneustes depressus especie nunca antes registrada para el Estado de Guerrero y fue recolectada durante el trabajo de campo (Tabla 2).

TABLA 1

Riqueza de equinodermos en isla La Roqueta de Acapulco, Guerrero, México, por clase y categorías taxonómicas

TABLE 1

Echinoderms richness on La Roqueta island of Acapulco, Guerrero, México, by class and their taxonomic categories

\begin{tabular}{lccccc} 
& Órdenes & Familias & Géneros & Especies & Representatividad número de especies \\
Asteroidea & 1 & 3 & 5 & 5 & $12 \%$ \\
Ophiuroidea & 1 & 4 & 5 & 14 & $35 \%$ \\
Echinoidea & 5 & 6 & 8 & 8 & $20 \%$ \\
Holothuroidea & 2 & 3 & 5 & 13 & $33 \%$ \\
Total & 9 & 16 & 23 & 40 & $100 \%$ \\
\hline
\end{tabular}


TABLA 2

Listado taxonómico de los equinodermos de isla La Roqueta, Acapulco, Guerrero, México

TABLE 2

Taxonomic list of the echinoderms of La Roqueta island, Acapulco, Guerrero, México

El arreglo taxonómico del presente listado está basado en el Catálogo de Autoridades Taxonómicas de los Equinodermos de México (Solís-Marín et al., 2017)

PHYLUM ECHINODERMATA De Bruguière, 1791

CLASE ASTEROIDEA de Blainville, 1830

ORDEN VALVATIDAPerrier, 1884

Familia Oreasteridae Fisher, 1911

Género Nidorellia Gray, 1840 Nidorellia armata (Gray, 1840)

Género Pentaceraster Döderlein, 1916 Pentaceraster cumingi (Gray, 1840)

Familia Mithrodiidae Viguier, 1878

Género Mithrodia Gray, 1840 Mithrodia bradleyi Verrill, 1870

Familia Ophidiasteridae Verrill, 1870

Género Pharia Gray, 1840 Pharia pyramidatus (Gray, 1840)

Género Phataria Gray, 1840 Phataria unifascialis (Gray, 1840)

CLASE OPHIUROIDEA Gray, 1840

ORDEN OPHIURIDA Müller \& Troschel, 1840

Familia Ophiocomidae Ljungman, 1867

Género Ophiocoma Agassiz, 1836 Ophiocoma aethiops Lütken, 1859

Género Ophiocomella Ophiocomella alexandri Lyman, 1860

Familia Ophiodermatidae Ljungman, 1867

Género Ophioderma Müller \& Troschel, 1840 Ophioderma panamensis Lütken, 1859 Ophioderma teres (Lyman, 1860) Ophioderma sp 1

Familia Ophiactidae Matsumoto, 1915

Género Ophiactis Lütken, 1856 Ophiactis sp 1

Familia Ophiotrichidae Ljungman, 1866

Género Ophiothrix Müller \& Troschel, 1840 Ophiothrix spiculata Le Conte, 1851 Ophiothrix rudis Lyman, 1874

CLASE ECHINOIDEA Leske, 1778

ORDEN CIDAROIDA Claus, 1880

Familia Cidaridae Gray, 1825

Género Eucidaris Pomel, 1883

Eucidaris thouarsii (L. Agassiz \& Désor, 1846)

ORDEN DIADEMATOIDA Duncan, 1889

Familia Diadematidae Gray, 1855

Género Diadema Gray, 1825

Diadema mexicanum A. Agassiz, 1863

Género Astropyga Gray, 1825
Astropyga pulvinata (Lamarck, 1816)

ORDEN CAMARODONTA Jackson, 1912

Familia Echinometridae Gray, 1825

Género Echinometra Gray, 1825

Echinometra vanbrunti A. Agassiz, 1863

Familia Toxopneustidae Troschel, 1872

Género Toxopneustes A. Agassiz, 1841 Toxopneustes roseus (A. Agassiz, 1863)

Género Tripneustes L. Agassiz, 1841b Tripneustes depressus A. Agassiz, 1863 *

ORDEN CASSIDULOIDA Claus, 1880

Familia Cassidulidae L. Agassiz \& Desor, 1847

Género Rhyncholampas A. Agassiz, 1869 Rhyncholampas pacificus (A. Agassiz, 1863)

ORDEN SPATANGOIDA Claus, 1876

Familia Brissidae Gray, 1855

Género Meoma Gray, 1851 Meoma ventricosa grandis Gray, 1851

CLASE HOLOTHUROIDEA Blainville, 1834 ORDEN DENDROCHIROTIDA Grube, 1840

Familia Sclerodactylidae Panning, 1949

Género Neothyone Deichmann, 1941 Neothyone gibber (Selenka, 1867)

Género Pachythyone Deichmann, 1941 Pachythyone lugubris (Deichmann, 1939) Cucumaria flamma Solís-Marín \& Laguarda-Figueras, 1999

Género Pseudocnus Panning, 1949 Pseudocnus californicus (Semper, 1868)

ORDEN ASPIDOCHIROTIDA Grube, 1840

Familia Holothuriidae Ludwig, 1894

Género Holothuria Linnaeus, 1767

Subgénero Cystipus Haacke, 1880 Holothuria (Cystipus) rigida (Selenka, 1867)

Holothuria (Halodeima) kefersteinii (Selenka, 1867)

Subgénero Semperothuria Deichmann, 1958 Holothuria (Semperothuria) imitans Ludwig, 1875

Subgénero Thymiosycia Pearson, 1914 Holothuria (Thymiosycia) arenicola Semper, 1868 Holothuria (Thymiosycia) impatiens (Forskål, 1775)

Familia Stichopodidae Haeckel, 1896

Género Isostichopus Deichmann, 1958 Isostichopus fuscus (Ludwig, 1874)

* especie de nuevo registro para Guerrero, México 


\section{DISCUSIÓN}

Se registra un total de 40 especies de equinodermos en la isla La Roqueta en Acapulco, Guerrero, México, 14 especies para la Clase Ophiuroidea, 13 para Holothuroidea, seguida con ocho especies para Echinoidea y cinco para Asteroidea, de un total de 214 ejemplares recolectados durante agosto y octubre 2018 y mayo 2019. El Catálogo de Equinodermos Recientes de México de la Colección Nacional de Equinodermos ICMyL-UNAM, contiene 96 especies del Estado de Guerrero, de las cuales 54 se registran para Bahías de Acapulco y 24 solo para la isla La Roqueta (Laguarda-Figueras, Solís-Marín, \& Caballero-Ochoa, 2017).

De acuerdo a los estudios de riqueza, abundancia y diversidad de ofiuroideos realizados por Hernández (2015) y García (2019) en diversos sitios de Bahías de Acapulco, registran seis y ocho especies de 303 y 462 ejemplares recolectados respectivamente en la isla $\mathrm{La}$ Roqueta, a diferencia del presente estudio con tan solo 214 ejemplares recolectados que corresponden a las cuatro clases presentes de equinodermos (Asteroidea, Ophiuroidea, Echinoidea y Holothuroidea) con 14 especies solo para la Clase Ophiuroidea.

Las 40 especies registradas con el actual estudio, 26 son nuevos reportes, que corresponde a diez especies de la Clase Ophiuroidea, siete de Holothuroidea, siete de Echinoidea y dos de la Clase Asteroidea. Estos 40 registros representan una valiosa aportación al conocimiento de la biodiversidad de la isla La Roqueta, lo que es relevante para la conservación y protección de este sitio como futura área natural protegida y de particular interés turístico en Guerrero y en México. Tripneustes depressus, conocido como erizo blanco o erizo café de la Clase Echinoidea, fue recolectado en agosto del 2018 y mayo 2019, esta especie representa un nuevo registro para la isla La Roqueta y para Guerrero.

Estudios en otras islas mexicanas, como los realizados por Padilla-Pérez et al., (2017), reconocen que las áreas insulares albergan alta biodiversidad, proporcionan los servicios ecosistémicos de soporte, regulación y suministro.
Sin embargo, muchas de las islas en México han generado gran interés turístico y en consecuencia altos niveles de visita, lo cual ha provocado una degradación en los ecosistemas bentónicos marinos, afectando a los organismos asociados a estos ecosistemas (Padilla-Pérez et al., 2017). Los estudios realizados sobre la biodiversidad de estas áreas insulares son de relevante importancia, contienen elementos únicos a diferencia de las áreas continentales. La generación de más estudios sobre su flora y fauna contribuyen a su conocimiento y a reconocer la función que tienen todas y cada una de las especies presentes en estos singulares hábitats. Para la isla La Roqueta en Acapulco, Guerrero, México, los resultados del presente estudio aportan información generada por una revisión bibliográfica, trabajo de campo y de laboratorio, que contribuye al conocimiento de la biodiversidad de equinodermos, con miras a un manejo adecuado para su conservación, además para futuros estudios sobre distribución, ecología, taxonomía, fisiología, sistemática molecular, entre otros, sobre los equinodermos y otras especies.

\section{AGRADECIMIENTOS}

La presente investigación se realizó con la colaboración de estudiantes, tesistas y prestadores de servicio social de Licenciatura en Biología de la Universidad Michoacana de San Nicolás de Hidalgo. Especial agradecimiento a quienes de manera incondicional apoyaron con su experiencia y conocimiento en las actividades de campo: Héctor Nava, Alejandro Montoya, Jesús Magaña y Alejandro Vega. Este trabajo no hubiera sido posible con las enseñanzas recibidas de Francisco Solís, Andrea Caballero, Carlos Conejeros y el apoyo del equipo de trabajo del ICMyL-UNAM.

\section{RESUMEN}

Introducción: Guerrero es el cuarto Estado más biodiverso en México, ejemplo de ello, es la riqueza de equinodermos habitantes en sus costas, alberga el mayor número de especies en comparación con los estados del Pacífico Sur Mexicano, presenta 135 especies, mientras 
que Oaxaca 94 y Chiapas 15. El presente estudio se desarrolló en isla La Roqueta en Acapulco, Guerrero, México, es parte del $0.3 \%$ del territorio nacional. Las islas son el hábitat de mayor número de especies y de endemismos, comparadas con áreas continentales de igual dimensión, isla La Roqueta, como otras islas en México, son reconocidas como sitios de gran valor, sin embargo, enfrentan graves amenazas que ponen en riesgo su estabilidad. Objetivo: Enlistar las especies de equinodermos en isla La Roqueta de Acapulco, Guerrero, México. Métodos: Se realizaron recolectas en agosto y octubre 2018 y mayo 2019, mediante buceo libre y Scuba en cuatro sitios, usando técnicas de captura directa, búsqueda de barrido, aleatorio y transecto. Resultados: 214 ejemplares fueron recolectados, en cuatro clases, nueve órdenes, 16 familias y 23 géneros, para un total de 40 especies. Se reconocen registros importantes: Ophiocoma sp., Phataria sp., Pentaceraster sp., Cucumaria sp., Astropyga sp., Meoma sp., Diadema sp., Afrocucumis ovulum, Pseudocnus californicus, Neocucumis veleronis y Holothuria imitans. Además, se destacan nuevos registros para el estado. Conclusiones: La complejidad de los diversos ambientes y sustratos marinos en Guerrero, en particular de isla La Roqueta, denota la posibilidad de nuevas especies.

Palabras clave: Echinodermata; riqueza; listado taxonómico; nuevo registro; isla La Roqueta; Guerrero; México.

\section{REFERENCIAS}

Aguirre-Muñoz, A., Bezaury-Creel, J.E., De la Cueva, H., March-Mifsut, I.J., Peters-Recagno, E., RojasGonzález de Castilla, S., \& Santos-del Prado Gasca, K. (2010). Islas de México, Un recurso estratégico. México: Instituto Nacional de Ecología (INE), The Nature Conservancy (TNC), Grupo de Ecología y Conservación de Islas, A.C. (GECI), Centro de Investigación Científica y de Educación Superior de Ensenada (CICESE).

Botello, F., Sánchez-Cordero, V., \& Ortega-Huerta, M.A. (2015). Disponibilidad de hábitats adecuados para especies de mamíferos a escalas regional (estado de Guerrero) y nacional (México). Revista Mexicana de Biodiversidad, 86, 226-237.

Caso, M.E. (1951). Contribución al conocimiento de los ofiuroideos de México. I. Algunas especies de ofiuroideos litorales. Anales del Instituto de Biología UNAM, 22(1), 219-312.

Caso, M.E. (1961). Los equinodermos de México (Tesis Doctoral). Universidad Nacional Autónoma de México, México.

Comisión Nacional de Áreas Naturales Protegidas (CONANP). (2018). Estudio Previo Justificativo para la modificación de la declaratoria de Santuarios de Playas Tortugueras. México: SEMARNAT, CONANP.
Conejeros-Vargas, C.A. (2015). Taxonomía y distribución de los equinodermos (Echinodermata) de las costas de Michoacán, México (Tesis de Licenciatura). Universidad Nacional Autónoma de México, México.

García, A.S.A. (2019). Riqueza, abundancia y diversidad de la Clase Ophiuroidea (Filo: Echinodermata) y su relación con factores exógenos, en Acapulco, Guerrero, México (Tesis de Maestría). Universidad Autónoma de Guerrero, México.

Granja-Fernández, R., Solís-Marín, F.A., Benítez-Villalobos, F., Herrero-Pérezrul, M.D., \& López-Pérez, A. (2015). Checklist of echinoderms (Echinodermata) from the Southern Mexican Pacific: a historical review. Revista de Biología Tropical, 63(2), 87-114.

Hernández, M.A. (2015). Riqueza, abundancia y diversidad de ofiuroideos (Echinodermata: Ophiuroidea) en cinco sitios rocosos de Acapulco, Guerrero, México (Tesis de Licenciatura). Universidad Autónoma de Guerrero, México.

Laguarda-Figueras, A., Solís-Marín, F.A., \& CaballeroOchoa, A.A. (2017). Actualización del Catálogo de los Equinodermos Recientes de México (Fase III). México: ICMyL, UNAM. Informe final SNIBCONABIO. Proyecto No. HA018.

Niño-Gutiérrez, N. (2008). Ambiente de la isla Roqueta de Acapulco, Guerrero, México. El Periplo Sustentable. Espacio de análisis y reflexión sobre Turismo Sustentable, 14, 5-25.

Niño-Gutiérrez, N. \& Pérez-Alcántara, B. (2011). Conservación y Gestión en la Unidad de Manejo Ambiental Mexicana La Roqueta. Revista Geográfica de América Central, Número Especial EGAL, 2, 1-13.

Padilla-Pérez, M.S., Rodríguez-Troncoso, A.P., SoteloCasas, R.C., \& Cupul-Magaña, A.L. (2017). Equinodermos del Parque Nacional Islas Marietas: Generalidades, importancia e identificación visual como herramienta para su protección. Áreas Naturales Protegidas Scripta, 3(2), 51-92.

Solís-Marín, F. A., Arriaga-Ochoa, J. A., Laguarda-Figueras, A., Frontana-Uribe, S. C., \& Durán-González, A. (2009). Holoturoideos (Echinodermata: Holothuroidea) del Golfo de California. México: Comisión Nacional para el Conocimiento y Uso de la Biodiversidad, ICMyL, UNAM.

Solís-Marín, F. A., Laguarda-Figueras, A., \& HoneyEscandón, M. (2014). Biodiversidad de equinodermos (Echinodermata) en México. Revista Mexicana de Biodiversidad, 85, 441-449.

Solís-Marín, F. A., Caballero-Ochoa, A. A., Frontana-Uribe, S. C., Laguarda-Figueras, A., \&, Durán-González, A. (2017). Catálogo de Autoridades Taxonómicas de los Equinodermos de México. México: ICMyL, UNAM. Informe final, SNIB-CONABIO, Proyecto No. Z002. 